\title{
Study on micro-damage model of clay under tension
}

\author{
Meng Cui ${ }^{1,2,3^{*}}$, Ting Yin ${ }^{1}$, Junjie Zheng ${ }^{2}$ and Xiao Fu ${ }^{3}$ \\ ${ }^{1}$ Nanchang Construction Engineering Group Company, Nanchang, JiangXi Province, 330038, China \\ ${ }^{2}$ School of Civil Engineering and Mechanics, Huazhong University of Science and Technology, Wuhan, HuBei Province, 430074, China \\ ${ }^{3}$ College of Civil and Structure Eng., Nanchang Inst. of Technology, Nanchang, JiangXi Province, 330099, China
}

\begin{abstract}
To improve the theoretical research on the tensile properties of soil, the damage model study was carried out in the present paper based on existing experimental results. In combination with the variation of the micro-structure of clay during the stretching process, the damage variable is defined by coupling the porosity with the pore distribution. According to the structure damage during the tensile process of clay under different initial conditions, the damage evolution equation is obtained and the damage model is established. At the same time, the applicability of the damage evolution equation and the damage model was verified by experimental data.
\end{abstract}

\section{Introduction}

With the continuous exploitation of underground space, the engineering problems caused by the tensile failure of the soil are increasingly prominent, e.g., the surface subsidence during the construction of the subway, the settlement basin or funnel in the excavation of the foundation pit. In addition, the tensile cracks at the top of the slope, the tensile cracks in the core wall of earth-rock dam, etc. are also caused by the tensile failure of the soil. Therefore, The tensile properties of soil have attracted more and more scholars' attention, and a series of related studies have also been carried out.

At present, the research on the tensile properties of soil is mainly based on experiments, which can be divided into two categories, i.e., direct stretching and indirect stretching, e.g., see [1-6]. Overview of the existing research results, they mainly focus on the test of tensile strength of soil under different conditions [7-11], and the evolution of micro-structure during tensile failure is discussed in a few papers [12]. However, the corresponding theoretical research is not deep. In the present paper, the damage mechanics model during tensile failure is studied based on the micro-experimental results, which were already carried out in reference [12].

\section{Damage variable}

The damage variable is the link between the structural characteristics and the physical and mechanical properties, and it is also the prerequisite for the quantitative analysis of the damage mechanics. In addition to the characteristics of material structure damage, the definition of the damage variable should generally consider the feasibility of parameter acquisition. The damage of soil during stretching causes micro-structural changes, so in the present paper the damage variable will be defined on a micro level. According to the experimental results which is shown in reference [12], and the related literature research, the change of pore morphology can better reflect structural damage compared to the change of particle morphology. In the present paper, two pore parameters are adopted to define the damage variable, i.e., the porosity and distribution fractal dimension of pore, and the damage variable is the linear coupling of the two parameters.

Assuming that the initial porosity of the sample is $\Phi_{0}$, and the porosity under tensile failure is $\Phi_{u}$, also the porosity under a certain state during stretching is $\Phi$, so the damage variable based on porosity can be defined as

$$
D_{1}=1-\frac{\Phi_{u}-\Phi}{\Phi_{u}-\Phi_{0}}
$$

The same principle, the damage variable based on the distribution fractal dimension of pore can be defined as

$$
D_{2}=1-\frac{D_{f u}-D_{f}}{D_{f u}-D_{f 0}}
$$

where $D_{f 0}, D_{f u}$ and $D_{f}$ are the distribution fractal dimension of pore under initial state, tensile failure and a certain state during stretching respectively.

The damage variable $D$ is defined as

$$
D=\frac{D_{1}+D_{2}}{2}=\left(\frac{\Phi-\Phi_{0}}{\Phi_{u}-\Phi_{0}}+\frac{D_{f}-D_{f 0}}{D_{f u}-D_{f 0}}\right) / 2
$$

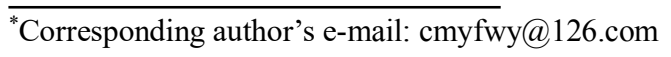




\section{Damage evolution equation}

The study of damage evolution equation includes the equation establishment, parameters determination and equation verification.

\subsection{Equation establishment}

According to Eq. (3) and test data in reference [12], the damage variable of six different initial condition samples during stretching is calculated and shown in figure 1, where symbol $\mathrm{C}$ represents the compaction of the samples, and symbol $\mathrm{w}$ represents the moisture content of the samples. It can be seen from Fig. 1 that the evolution of damage variable is basically the same, which can be divided into two stages. At the same time, combined with macro tensile test results in reference [6], the stress at the demarcation point of two stages is the peak stress. As shown in Fig. 1, $\varepsilon_{f}$ is the demarcation point of the sample with $\mathrm{C}=100 \%$ and $\mathrm{w}=21.5 \%$. By curve fitting, the evolution equation can be established.

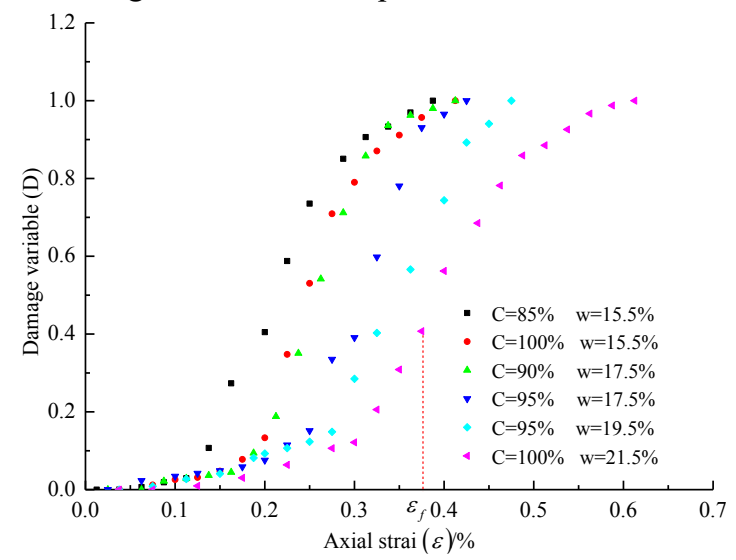

Figure 1. Damage variable-axial strain scatter plot of samples with different initial conditions

When $0 \leq \varepsilon \leq \varepsilon_{f}$, the general form of evolution equation is

$$
D=a\left(\varepsilon / \varepsilon_{f}\right)^{n}
$$

where $\mathbf{a}$ and $\mathbf{n}$ are material constants.

When $\varepsilon>\varepsilon_{f}$, the form of evolution equation is

$$
D=1-\frac{b}{c\left(\varepsilon / \varepsilon_{f}-1\right)^{m}+\left(\varepsilon / \varepsilon_{f}\right)}
$$

where $\mathbf{c}$ and $\mathbf{m}$ are curve constants.

So, the damage evolution equation can be expressed as

$$
D= \begin{cases}a\left(\varepsilon / \varepsilon_{f}\right)^{n} & 0 \leq \varepsilon \leq \varepsilon_{f} \\ 1-\frac{b}{c\left(\varepsilon / \varepsilon_{f}-1\right)^{m}+\varepsilon / \varepsilon_{f}} & \varepsilon>\varepsilon_{f}\end{cases}
$$

\subsection{Parameter determination}

When $\varepsilon=\varepsilon_{f}$, two boundary conditions can be expressed as

$$
\begin{aligned}
& \left.\sigma_{t}\right|_{\varepsilon=\varepsilon_{f}}=\sigma_{t f} \\
& \left.\frac{d \sigma_{t}}{d \varepsilon}\right|_{\varepsilon=\varepsilon_{f}}=0
\end{aligned}
$$

Meanwhile, the relationship between tensile stress and damage variable is

$$
\sigma_{t}=E_{t}(1-D) \varepsilon
$$

where $E_{t}$ is the initial elastic modulus of the sample.

So, when $\varepsilon=\varepsilon_{f}$, the relationship is

$$
\sigma_{t f}=E_{t}(1-a) \varepsilon_{f}
$$

let $E_{t f}{ }^{\prime}=\frac{\sigma_{t f}}{\varepsilon_{f}}$, a can be expressed as

$$
a=1-\frac{E_{t f}^{\prime}}{E_{t}}
$$

Meanwhile, according to Eq. (6), (8) and (9), $\mathbf{n}$ can be expressed as

$$
n=\frac{1-a}{a}
$$

when $\varepsilon=\varepsilon_{f}$, the curve is continuous, so $\mathbf{b}$ can be expressed as

$$
b=1-a
$$

In addition, $\mathbf{c}$ and $\mathbf{m}$ are curve constants, which can be obtained from two sets of test data.

\subsection{Equation verification}

In order to verify the applicability of the evolution equation, two samples with different initial conditions are selected, i.e., the sample with $\mathrm{C}=100 \%, \mathrm{w}=21.5 \%$ and the sample with $\mathrm{C}=90 \%, \mathrm{w}=17.5 \%$. For the first sample, by the equations in 3.2 the constants can be calculated as $a=0.24, n=3.2, b=0.76, c=9.8$ and $m=1.7$. Same with the second sample, the constants are $a=0.19, n=4.2, b=0.81, c=19$ and $m=2.1$.The verification curves of the two samples are shown in figure 2 . From figure 2 , we can conclude that the theoretical results calculated by the damage evolution equation are in good agreement with the experimental results, and the deviation can be controlled within $5 \%$. 


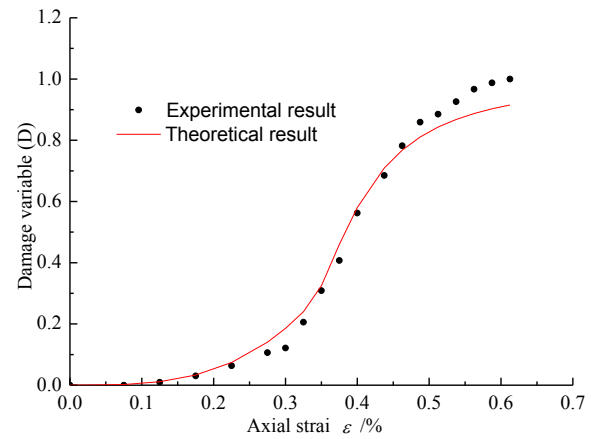

(a) $\mathrm{C}=100 \%, \mathrm{w}=21.5 \%$

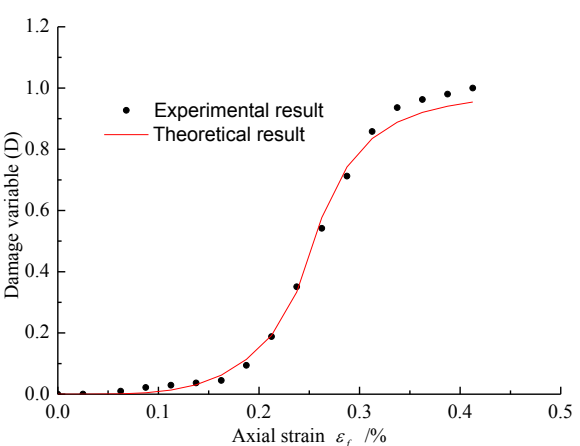

(b) $\mathrm{C}=90 \%, \mathrm{w}=17.5 \%$

Figure 2 . The verification curves of damage evolution equation

\section{Damage constitutive model}

The study of damage constitutive model includes the model establishment and model verification.

\subsection{Model establishment}

Through comparative analysis, the damage constitutive model based on strain equivalence hypothesis has better applicability to clay stretching, which can be expressed as

$$
\sigma=\tilde{\sigma}(1-D)=\tilde{E}(\varepsilon) \varepsilon=(1-D) E \varepsilon
$$

where $\tilde{\sigma}$ is effective stress, $D$ is damage variable, $\tilde{E}(\varepsilon)$ is elastic modulus under a certain strain state during damage.

Substituting the Eq. (6) into the Eq. (14), the expression of the damage constitutive model of the clay tensile failure is

$$
\sigma_{t}= \begin{cases}{\left[1-a\left(\varepsilon / \varepsilon_{f}\right)^{n}\right] E_{t} \varepsilon} & 0 \leq \varepsilon \leq \varepsilon_{f} \\ \frac{b}{c\left(\varepsilon / \varepsilon_{f}-1\right)^{m}+\varepsilon / \varepsilon_{f}} E_{t} \varepsilon & \varepsilon>\varepsilon_{f}\end{cases}
$$

All the parameters in Eq. (15) are the same as mentioned above.

\subsection{Model verification}

In order to verify the applicability of the damage constitutive model, four samples with different initial conditions are selected, i.e., the sample with $\mathrm{C}=95 \%$, $\mathrm{w}=17.5 \%$ (No.1), sample with $\mathrm{C}=95 \%, \mathrm{w}=19.5 \%$ (No.2), sample with $\mathrm{C}=100 \%, \mathrm{w}=21.5 \%$ (No.3) and the sample with $\mathrm{C}=100 \%, \mathrm{w}=15.5 \%$ (No.4). The tensile stress-axial strain curves of damage constitutive model and that of experimental results are shown in figure3, where the experimental results are cited in reference [6]. From figure 3 , we can conclude that the suitability of damage constitutive model is good before peak stress, but there is a certain deviation after the peak stress, which is due to the non-pure brittle failure of the clay.

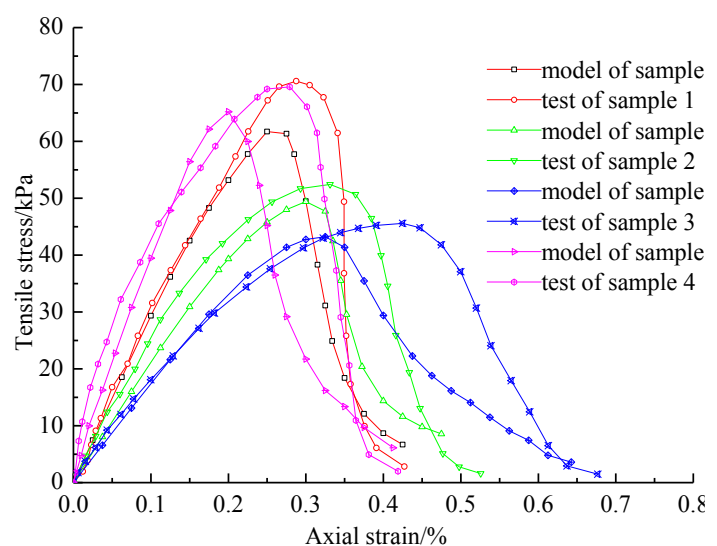

Figure 3. The verification curves of damage constitutive model

\section{Concluding remarks}

In this work, the damage variable is defined by the porosity and the distribution fractal dimension of pore from the microscopic level. According to the evolution law of the damage variable, which is based on the microscopic tensile failure test of clay, the damage evolution equation is established, and the applicability of the evolution equation is verified by two sets of examples. It is found that the damage evolution equation match well with the test results. Also, the damage constitutive model based on strain equivalence hypothesis is established, and by four sets of examples the applicability of the damage constitutive model is verified. It shows that the damage constitutive model match well before peak stress, but not so good after peak stress, which is due to the non-pure brittle failure of clay. Overall, the proposed damage model can reflect the evolution of structural damage during stretching.

\section{Acknowledgements}

The present work was supported by the National Natural Science Foundation of China (Grant No.51609114) and the Science and Technology Research Program of Jiangxi Provincial Education Department, China (Grant No. GJJ180929). 


\section{References}

1. Lu N, Wu B, Tan C P. (2007) Tensile strength characteristics of unsaturated sands. Journal of Geotechnical and Geoenvironmental Engineering, 133:144-154.

2. Mesbah A, Morel J C, Walker P. (2004) Development of a direct tensile test for compacted earth blocks reinforced with natural fibers. Journal of Materials in Civil Engineering, 16:95-98.

3. Nahlawi H, Chakrabarti S, Kodikara J. (2004) A direct tensile strength testing method for unsaturated geomaterials. Geotechnical Testing Journal, 27:1-6.

4. Consoli N C, De Moraes R R, Festugato L. (2013) Parameters controlling tensile and compressive strength of fiber-reinforced cemented soil. Journal of Materials in Civil Engineering, 25: 1568-1573.

5. Li H D, Tang C S, Xu Q L. (2016) Advances in experimental testing methods of soil tensile strength. Rock and Soil Mechanics, 37:175-186.

6. Cui M, Han S Y, Hong B N. (2017) Development and application of a new geotechnical device for direct tension test. Rock and Soil Mechanics, 38:1832- 1840.

7. Azmatch T F, Sego D C, Arenson L U. (2011) Tensile strength and stress-strain behavior of Devon silt under frozen fringe conditions. Cold Regions Science and Technology, 68: 85-90.

8. Tang C, Pei X, Wang D. (2015) Tensile strength of compacted clayey soil. Journal of Geotechnical and Geoenvironmental Engineering, 141:1-8.

9. Lv H B, Zeng Z T, Ge R D. (2013) Experimental study of tensile strength of swell-shrink soils. Rock and Soil Mechanics, 34:615- 620.

10. Satoshi A, Kohei N. (2009) Tensile strength of frozen soil in the temperature range of the frozen fringe. Cold Regions Science and Technology, 57:13- 22.

11. Tang G X, Graham J. (2000) A method for testing tensile strength in unsaturated soils. Geotechnical Testing Journal, 23:377-382.

12. Cui M, Liu J, Han S Y, Hong B N. (2018) Development and application of microstructure change test system in the process of soil tensile failure. Rock and Soil Mechanics, 39:4278-4286. 